\title{
Alfvén wave propagation and dissipation in a 3D-structured compressible plasma
}

\author{
F. Malara ${ }^{1,2}$, M. F. De Franceschis ${ }^{1,2}$, and P. Veltri ${ }^{1,2}$ \\ 1 Dipartimento di Fisica, Università della Calabria, via P. Bucci, 87036 Rende (CS), Italy \\ 2 Istituto Nazionale per la Fisica della Materia, Unità di Cosenza, via P. Bucci, 87036 Rende, Italy
}

Received 2 June 2003 / Accepted 30 July 2003

\begin{abstract}
The propagation and the dissipation of small-amplitude Alfvén waves in an equilibrium configuration characterized by three-dimensional inhomogeneities is investigated. Disturbances are supposed to have a typical wavelength smaller than the scale of nonuniformity of the background structure, which allows us to use a WKB expansion technique. The approach we used is similar to that employed by Petkaki et al. (1998), who studied the case of an incompressible plasma. In the present paper a compressible cold plasma is considered, which is more suitable than an incompressible plasma to describe the situation of the solar Corona, where $\beta \ll 1$. Small wavelengths allow to decouple Alfvén from magnetosonic fluctuations at the linear level. Considering small Alfvénic wavepackets, the evolution equations for the position, wavevector, and energy are derived. These equations are similar to those obtained by Petkaki et al. (1998), and they have the same form only if the background density $\rho^{(0)}$ is supposed to be constant. Then, the results found by Petkaki et al. (1998) for an incompressible plasma are valid also in a compressible cold plasma, provided that $\rho^{(0)}=$ const.: in particular, in the presence of regions of chaotic fieldlines the wavevector of Alfvénic perturbations exponentially grows and the dissipation time of the wave is $t_{\mathrm{d}} \propto \log S, S$ being the Reynolds number. Thus, a fast dissipation is attained even with large values of $S$, as in the Corona. The equations derived in the present paper are more general than those of Petkaki et al. (1998), since they can be used also with a nonuniform background density. The present model is discussed with reference to the problem of coronal heating.
\end{abstract}

Key words. magnetohydrodynamics (MHD) - waves - Sun: corona

\section{Introduction}

Dissipation of Alfvén waves represents one of the most studied mechanisms which has been invoked to explain the nonradiative heating of astrophysical plasmas, like the solar Corona and the solar wind. In such plasmas, which are permeated by a strong magnetic field, Alfvén waves can transfer both mechanical and electromagnetic energy from distant parts. Alfvénic fluctuations, which have been directly detected in the solar wind (Belcher \& Davis 1971) as the main fluctuation component, are probably present also in the Corona, from where solar wind is emanated. Due to the small dissipative coefficients (resistivity and relevant viscosity), one important point in these theories is how to dissipate Alfvén waves before they leave the system. The presence of inhomogeneities in the background structure represents an important ingredient, since interactions between waves and background nonuniformities can move the wave energy to small scales, where it can be efficiently dissipated. In this framework, we are not interested to the specific dissipation mechanism; rather, we focus on the dynamical processes which generate small scales in the wave structure. In a $2 \mathrm{D}$ inhomogeneous structure, where the Alfvén velocity $c_{\mathrm{A}}$ varies in a direction perpendicular to the magnetic field, two mechanisms have been extensively investigated: (i) phase-mixing, due to differences of phase velocity in the direction transverse to wave propagation, which progressively bends wavefronts; and (ii) resonant absorption, which concentrates the wave energy in a thin layer where the frequency locally matches a characteristic frequency (Alfvén or cusp frequency). These mechanisms have been studied both by searching for normal modes of the inhomogeneous structure (Kappraff \& Tataronis 1977; Mok \& Einaudi 1985; Steinolfson 1985; Davila 1987; Hollweg 1987a,b; Califano et al. 1990; Davila 1991; Califano et al. 1992); and by considering the time evolution of an initial disturbance (Lee \& Roberts 1986; Malara et al. 1992, 1996). Effects of density stratification and magnetic line divergence (Ruderman et al. 1998), as well as nonlinear coupling with compressive modes (Nakariakov et al. 1997, 1998) have also been considered. Recently, the evolution of 3D localized pulses propagating in a 1D-inhomogeneous equilibrium structure has been studied (Tsiklauri \& Nakariakov 2002; Tsiklauri et al. 2003), considering the effects of phase-mixing in the presence of both an Alfvénic and a magnetosonic component.

Send offprint requests to: F. Malara, e-mail: malara@fis.unical.it 
Alfvén wave propagation in three dimensional magnetic structures have been considered by Similon \& Sudan (1989). In this case the background magnetic field can have a chaotic behavior: there can be regions where initially nearby magnetic lines move apart exponentially (Zimbardo et al. 1984, 1995). Since Alfvén waves locally follows magnetic lines, this results in a stretching of the wave pattern and a consequent formation of small scales, which takes place exponentially in time. As a consequence, the dissipation time $t_{\mathrm{d}}$ follows the scaling law (Similon \& Sudan 1989):

$t_{\mathrm{d}} \propto \log S$

where $S$ is the relevant viscous and/or resistive Reynolds number. Thus, dissipation time can remain relatively small even for large values of the Reynolds number $S$, as in the coronal plasma. The mechanism proposed by Similon \& Sudan (1989) has been investigated by Petkaki et al. (1998) (Paper I) and by Malara et al. (2000). These authors built up a model, based on a WKB expansion of incompressible magnetohydrodynamic (MHD) equations, in which propagation and dissipation of Alfvénic wavepackets in 3D magnetic fields is described. From that model they found that in regions where magnetic lines are chaotic a fast dissipation of wavepackets takes place, which follows the scaling law (1). This effect can co-exist with a slower dissipation determined by phase-mixing, in regions where the magnetic topology is quasi-2D.

One important drawback of the model of Paper I is the assumption of incompressibility. Actually, incompressibility is recovered in the limit $\beta \rightarrow \infty$, with $\beta=p / p_{\mathrm{M}}$ ( $p$ is the gas pressure and $p_{\mathrm{M}}=B^{2} / 8 \pi$ is the magnetic pressure). On the contrary, the plasma in the Corona is dominated by the magnetic field, so that $\beta \sim 10^{-2} \ll 1$. Thus, a low- $\beta$ assumption instead of incompressibility would be more suitable to describe the coronal conditions. In the present paper we will consider the propagation and dissipation of fluctuations in 3D magnetic equilibrium structures, in a cold plasma, i.e., in the limit $\beta \rightarrow 0$. Contrary to Paper I, compressive fluctuations can now propagate, along with Alfvén waves. We will derive the equations describing the time evolution of Alfvénic wavepackets, and we will show that this equations have a form similar to that found for an incompressible plasma. All the results found in the incompressible case by the model of Paper I are valid also for a cold plasma, which better represents the conditions of the solar Corona, provided that a uniform background density is assumed. However, the equations which will be derived are more general, since they can be used even in presence of an inhomogeneous background density, as in the solar Corona.

\section{Derivation of the model}

The model is intended to describe the time evolution and dissipation of Alfvénic perturbations. As discussed in the previous section, we include compressibility of the plasma, but we neglect both the gradient of gas pressure and gravity with respect to the Laplace force $\boldsymbol{j} \times \boldsymbol{B} / \boldsymbol{c}$ (cold plasma). Using dimensionless variables, the MHD equations can be written in the following form

$$
\frac{\partial \rho}{\partial t}+v_{n} \frac{\partial \rho}{\partial x_{n}}+\rho \frac{\partial v_{n}}{\partial x_{n}}=0
$$

$\rho\left[\frac{\partial v_{i}}{\partial t}+v_{n} \frac{\partial v_{i}}{\partial x_{n}}\right]=-\frac{\partial}{\partial x_{i}}\left(\frac{B_{n} B_{n}}{8 \pi}\right)+\frac{1}{4 \pi} B_{n} \frac{\partial B_{i}}{\partial x_{n}}+\eta_{\mathrm{N}} \frac{\partial^{2} v_{i}}{\partial x_{n} \partial x_{n}}+\left(\zeta_{\mathrm{N}}+\frac{\eta_{\mathrm{N}}}{3}\right) \frac{\partial}{\partial x_{i}} \frac{\partial v_{n}}{\partial x_{n}}$

$\frac{\partial B_{i}}{\partial t}+v_{n} \frac{\partial B_{i}}{\partial x_{n}}=B_{n} \frac{\partial v_{i}}{\partial x_{n}}-B_{i} \frac{\partial v_{n}}{\partial x_{n}}+\lambda_{\mathrm{N}} \frac{\partial^{2} B_{i}}{\partial x_{n} \partial x_{n}}$

In these equations lengths have been normalized to a characteristic length $L$, which represents the typical scale over which the equilibrium structure varies. The magnetic field is normalized to a characteristic magnetic field $B_{0}$, the density to a characteristic density $\rho_{0}$, the velocity to the corresponding Alfvén velocity $c_{\mathrm{A} 0}=B_{0} /\left(4 \pi \rho_{0}\right)^{1 / 2}$, and the time to the Alfvén time $t_{\mathrm{A}}=L / c_{\mathrm{A} 0}$. The dimensionless dissipative coefficients are $\eta_{\mathrm{N}}=\eta / \rho_{0} L c_{\mathrm{A} 0}, \zeta_{\mathrm{N}}=\zeta / \rho_{0} L c_{\mathrm{A} 0}$, and $\lambda_{\mathrm{N}}=\lambda / L c_{\mathrm{A} 0}$, where $\eta$ and $\zeta$ are the viscosity coefficients and $\lambda$ is the magnetic diffusivity, which are assumed to be constant. Summation over dummy indices is hereafter understood.

We consider a small amplitude perturbation which propagates in an inhomogeneous equilibrium configuration. Density, velocity and magnetic field are written in the following form:

$$
\begin{aligned}
& \rho(\boldsymbol{x}, t)=\rho^{(0)}(\boldsymbol{x})+\varepsilon \rho^{(1)}(\boldsymbol{x}, t) \\
& \boldsymbol{v}(\boldsymbol{x}, t)=\boldsymbol{\varepsilon} \boldsymbol{v}^{(1)}(\boldsymbol{x}, t) \\
& \boldsymbol{B}(\boldsymbol{x}, t)=\boldsymbol{B}^{(0)}(\boldsymbol{x})+\varepsilon \boldsymbol{B}^{(1)}(\boldsymbol{x}, t)
\end{aligned}
$$

where $\varepsilon \ll 1$ represents the perturbation amplitude. The upper indices "(0)" and "(1)" denotes quantities relative to the equilibrium and to the perturbation, respectively. We assume that equilibrium quantities $\rho^{(0)}(\boldsymbol{x})$ and $\boldsymbol{B}^{(0)}(\boldsymbol{x})$ vary on the normalization length, which is $l=1$ in normalized units. The velocity at the order $\varepsilon^{0}$ is vanishing, corresponding to a static structure. 
An expansion of Eqs. (2)-(4) is carried out with respect to the small parameter $\varepsilon$. At the lowest order $O\left(\varepsilon^{0}\right)$ Eq. (2) is identically satisfied, while Eq. (3) gives the force-free condition

$\left(\nabla \times \boldsymbol{B}^{(0)}\right) \times \boldsymbol{B}^{(0)}=0$

which must be fulfilled by the equilibrium magnetic field. Equation (4) gives the condition $\lambda_{N} \nabla^{2} \boldsymbol{B}^{(0)}=0$. We assume that this equation is approximately satisfied in the limit of small $\lambda_{\mathrm{N}}$. This corresponds to neglecting the diffusion of the equilibrium magnetic field $\boldsymbol{B}^{(0)}$ with respect to faster phenomena, like wave propagation and dissipation. The condition $\lambda_{\mathrm{N}} \ll 1$ will be better specified later.

At the order $O\left(\varepsilon^{1}\right)$, we get the equations:

$\frac{\partial \rho^{(1)}}{\partial t}+v_{n}^{(1)} \frac{\partial \rho^{(0)}}{\partial x_{n}}+\rho^{(0)} \frac{\partial v_{n}^{(1)}}{\partial x_{n}}=0$

$\rho^{(0)} \frac{\partial v_{i}^{(1)}}{\partial t}=-\frac{\partial}{\partial x_{i}}\left(\frac{B_{n}^{(0)} B_{n}^{(1)}}{4 \pi}\right)+\frac{B_{n}^{(0)}}{4 \pi} \frac{\partial B_{i}^{(1)}}{\partial x_{n}}+\frac{B_{n}^{(1)}}{4 \pi} \frac{\partial B_{i}^{(0)}}{\partial x_{n}}+\eta_{\mathrm{N}} \frac{\partial^{2} v_{i}^{(1)}}{\partial x_{n} \partial x_{n}}+\left(\zeta_{\mathrm{N}}+\frac{\eta}{3}\right) \frac{\partial}{\partial x_{i}}\left(\frac{\partial v_{n}^{(1)}}{\partial x_{n}}\right)$

$\frac{\partial B_{i}^{(1)}}{\partial t}+v_{n}^{(1)} \frac{\partial B_{i}^{(0)}}{\partial x_{n}}=B_{n}^{(0)} \frac{\partial v_{i}^{(1)}}{\partial x_{n}}-B_{i}^{(0)} \frac{\partial v_{n}^{(1)}}{\partial x_{n}}+\lambda_{\mathrm{N}} \frac{\partial^{2} B_{i}^{(1)}}{\partial x_{n} \partial x_{n}}$

which will be used to describe the evolution of perturbations.

Another important assumption is that the scale length of perturbations keeps much smaller than that of the equilibrium structure, which is equal to 1 in dimensionless units:

$\delta \ll \ell \ll 1$

where $\delta$ is the characteristic wavelength of perturbation and $\ell$ is an intermediate scale. During the evolution, the wavelength tends to decrease in time (Paper I); thus, the condition (12) is always fulfilled, provided that it is satisfied at the initial time. The assumption (12) allows us to perform a WKB expansion of Eqs. (9)-(11), using the wavelength $\delta$ as expansion parameter. Due to the linear character of Eqs. (9)-(11), the perturbation is considered as a superposition of different modes, propagating independently from one another. Thus, perturbed quantities are expanded up to the first order in the parameter $\delta$ :

$\rho^{(1)}=\mathfrak{R}\left[\sum_{\alpha}\left(\rho_{0}^{\alpha}+\delta \rho_{1}^{\alpha}\right) \exp \left(i \frac{S^{\alpha}}{\delta}\right)\right]$

$v_{i}^{(1)}=\mathfrak{R}\left[\sum_{\alpha}\left(v_{0 i}^{\alpha}+\delta v_{1 i}^{\alpha}\right) \exp \left(i \frac{S^{\alpha}}{\delta}\right)\right]$

$B_{i}^{(1)}=\mathfrak{R}\left[\sum_{\alpha}\left(B_{0 i}^{\alpha}+\delta B_{1 i}^{\alpha}\right) \exp \left(i \frac{S^{\alpha}}{\delta}\right)\right]$

where " $\alpha$ " indicates the different propagating modes, the quantity $S^{\alpha}(\boldsymbol{x}, t)$ represents the phase of the $\alpha$ th mode, and the lower indices " 0 " and " 1 " indicate $O\left(\delta^{0}\right)$ quantities and $O\left(\delta^{1}\right)$ quantities, respectively. The phase $S^{\alpha}(x, t)$ is a real function, while all the other quantities in the expressions (13)-(15) are complex functions. $\mathfrak{R}(f)$ is the real part of a complex quantity $f$. In the following we will omit the symbol " $R$ ", working directly with the corresponding complex quantities: this is possible in consequence of the linearity of the evolution Eqs. (9)-(11). The frequency $\omega^{\alpha}$ and the wavevector $\boldsymbol{k}^{\alpha}$ of the $\alpha$ th mode depend both on $\boldsymbol{x}$ and $t$. They are defined by

$\omega^{\alpha}(\boldsymbol{x}, t)=-\frac{1}{\delta} \frac{\partial S^{\alpha}}{\partial t}=\frac{\Omega^{\alpha}(\boldsymbol{x}, t)}{\delta}$

$k_{n}^{\alpha}(\boldsymbol{x}, t)=\frac{1}{\delta} \frac{\partial S^{\alpha}}{\partial x_{n}}=\frac{K_{n}^{\alpha}(\boldsymbol{x}, t)}{\delta}$.

The quantities $\Omega^{\alpha}$ and $\boldsymbol{K}^{\alpha}$ are the renormalized order $O\left(\delta^{0}\right)$ frequency and wavevector, respectively, while both $\omega^{\alpha}$ and $\boldsymbol{k}^{\alpha}$ are of order $O\left(\delta^{-1}\right)$. It is worth noting that perturbation amplitudes $\rho_{0}^{\alpha}, \rho_{1}^{\alpha}, \boldsymbol{v}_{0}^{\alpha}, \boldsymbol{v}_{1}^{\alpha}, \boldsymbol{B}_{0}^{\alpha}$, and $\boldsymbol{B}_{1}^{\alpha}$, as well as the frequency $\Omega^{\alpha}$ and the wavevector $\boldsymbol{K}^{\alpha}$ are assumed to vary on the scale $l$. On the contrary, the exponential factor $\exp \left(i S^{\alpha} / \delta\right)$ varies over the scale $\delta$.

Finally, dissipative coefficients are supposed to be small quantities. It is necessary to specify their ordering with respect to the expansion parameter $\delta$. We will assume that dissipation coefficients are quantities of order $O\left(\delta^{2}\right)$. Then, we write

$\eta_{\mathrm{N}}=\tilde{\eta}_{\mathrm{N}} \delta^{2}, \zeta_{\mathrm{N}}=\tilde{\zeta}_{\mathrm{N}} \delta^{2}, \lambda_{\mathrm{N}}=\tilde{\lambda}_{\mathrm{N}} \delta^{2}$

where $\tilde{\eta}_{\mathrm{N}}, \tilde{\zeta}_{\mathrm{N}}$, and $\tilde{\lambda}_{\mathrm{N}}$ are order $O\left(\delta^{0}\right)$ quantities. With this assumption dissipative coefficients do not affect the wave propagation, but they enter in the equation of energy determining the wave dissipation. 


\section{1. $\operatorname{Order} \delta^{-1}$}

The expressions (13)-(15) and (18) are inserted into the Eqs. (9)-(11), which are expanded in powers of the parameter $\delta$. At the lowest order $O\left(\delta^{-1}\right)$ the following equation is obtained from Eq. (9):

$\sum_{\alpha}\left[-i \rho_{0}^{\alpha} \Omega^{\alpha}+i \rho^{(0)} v_{0 n}^{\alpha} K_{n}^{\alpha}\right] \exp \left(i \frac{S^{\alpha}}{\delta}\right)=0$.

We multiply this equation by the factor $\exp \left(-i S^{\beta} / \delta\right)$ and average over the intermediate scale $\ell$. Since the terms in square brackets in Eq. (19) vary on the larger scale $l$, we obtain

$\sum_{\alpha}\left[-i \rho_{0}^{\alpha} \Omega^{\alpha}+i \rho^{(0)} v_{0 n}^{\alpha} K_{n}^{\alpha}\right]\left\langle\exp \left(i \frac{S^{\alpha}-S^{\beta}}{\delta}\right)\right\rangle_{\ell}=0$

where angular brackets indicate an average over the intermediate scale $\ell$. The phases $S^{\alpha}$ of different modes are different, thus, the function $\exp \left[i\left(S^{\alpha}-S^{\beta}\right) / \delta\right]$ oscillates over the scale $\delta$, and its average value over the scale $\ell$ is vanishing, provided that $\alpha \neq \beta$. In other words

$\left\langle\exp \left( \pm i \frac{S^{\alpha}-S^{\beta}}{\delta}\right)\right\rangle_{\ell}=\delta_{\alpha \beta}$.

Using this condition, the Eq. (20) takes the form

$\rho_{0}^{\alpha} \Omega^{\alpha}-\rho^{(0)} v_{0 n}^{\alpha} K_{n}^{\alpha}=0$.

Applying the same procedure to Eqs. (10) and (11), at the order $O\left(\delta^{-1}\right)$ we obtain, respectively:

$v_{0 i}^{\alpha} \Omega^{\alpha}-\frac{B_{n}^{(0)}}{4 \pi \rho^{(0)}}\left(B_{0 n}^{\alpha} K_{i}^{\alpha}-B_{0 i}^{\alpha} K_{n}^{\alpha}\right)=0$

$B_{0 i}^{\alpha} \Omega^{\alpha}-\left(B_{i}^{(0)} v_{0 n}^{\alpha}-B_{n}^{(0)} v_{0 i}^{\alpha}\right) K_{n}^{\alpha}=0$

The Eqs. (22)-(24) allow us to calculate some properties of the solution at the order $O\left(\delta^{-1}\right)$. It is worth noting that quantities relative to a single mode appears in Eqs. (22)-(24); so, they can be solved for each mode, separately. In other words, each mode is decoupled from the others.

It is useful to define a local Cartesian reference frame, whose axes are indicated by $\{X, Y, Z\}$. The $X$-axis is parallel to $\boldsymbol{B}^{(0)}$, while the wavevector $\boldsymbol{K}^{\alpha}$ is in the $X Y$ plane. This reference frame is illustrated in Fig. 1.

We indicate by $\theta^{\alpha}$ the angle between $\boldsymbol{K}^{\alpha}$ and $\boldsymbol{B}^{(0)}$. Since both $\boldsymbol{K}^{\alpha}$ and $\boldsymbol{B}^{(0)}$ are functions of position, the orientation of these axes changes from point to point; however, such variations take place at the scale $l$ of the equilibrium structure. We can re-write the Eqs. (22)-(24) using the components of vector quantities $\left(\boldsymbol{B}^{(0)}, \boldsymbol{K}^{\alpha}, \boldsymbol{v}_{0}^{\alpha}\right.$ and $\left.\boldsymbol{B}_{0}^{\alpha}\right)$ with respect to the axes $X, Y$, and $Z$. The $X$ component of Eq. (23) reduces to $v_{0 X}^{\alpha} \Omega^{\alpha}=0$, which implies

$v_{0 X}^{\alpha}=0$

provided that $\Omega^{\alpha} \neq 0$. Thus, the perturbation velocity component parallel to $\boldsymbol{B}^{(0)}$ is vanishing, for propagating modes. In fact, in a cold plasma the only force acting on the fluid is the Laplace force $\boldsymbol{f}_{L}=\boldsymbol{j} \times \boldsymbol{B} / \boldsymbol{c}$, with $\boldsymbol{j}$ the current density, which vanishes for the equilibrium magnetic field. At the order $O\left(\varepsilon^{1}\right)$, the force associated with perturbations is $\boldsymbol{f}^{(1)}=\left(\boldsymbol{j}^{(1)} \times \boldsymbol{B}^{(0)}\right) / c+\left(\boldsymbol{j}^{(0)} \times \boldsymbol{B}^{(1)}\right) / c$. Since $\boldsymbol{j}^{(0)}$ is parallel to $\boldsymbol{B}^{(0)}$, the force $\boldsymbol{f}^{(1)}$ has no components in the direction of the equilibrium magnetic field $\boldsymbol{B}^{(0)}$. This explains the result of Eq. (25).

The Eq. (22), the $Y$ and $Z$ components of Eq. (23), and the Eq. (24) can be written in the following form

$A_{i j} U_{0 j}^{\alpha}=\Omega^{\alpha} U_{0 i}^{\alpha}$

where $\boldsymbol{U}_{0}^{\alpha}=\left(\rho_{0}^{\alpha}, B_{0 X}^{\alpha}, v_{0 Y}^{\alpha}, B_{0 Y}^{\alpha}, v_{0 Z}^{\alpha}, B_{0 Z}^{\alpha}\right)$ is the vector of unknowns, and $A_{i j}$ are the elements of the following matrix:

$A=\left(\begin{array}{cccccc}0 & 0 & \rho^{(0)} K^{\alpha} \sin \theta^{\alpha} & 0 & 0 & 0 \\ 0 & 0 & B^{(0)} K^{\alpha} \sin \theta^{\alpha} & 0 & 0 & 0 \\ 0 & \frac{B^{(0)} K^{\alpha} \sin \theta^{\alpha}}{4 \pi \rho^{(0)}} & 0 & -\frac{B^{(0)} K^{\alpha} \cos \theta^{\alpha}}{4 \pi \rho^{(0)}} & 0 & 0 \\ 0 & 0 & -B^{(0)} K^{\alpha} \cos \theta^{\alpha} & 0 & 0 & 0 \\ 0 & 0 & 0 & 0 & 0 & -\frac{B^{(0)} K^{\alpha} \cos \theta^{\alpha}}{4 \pi \rho^{(0)}} \\ 0 & 0 & 0 & 0 & -B^{(0)} K^{\alpha} \cos \theta^{\alpha} & 0\end{array}\right)$. 


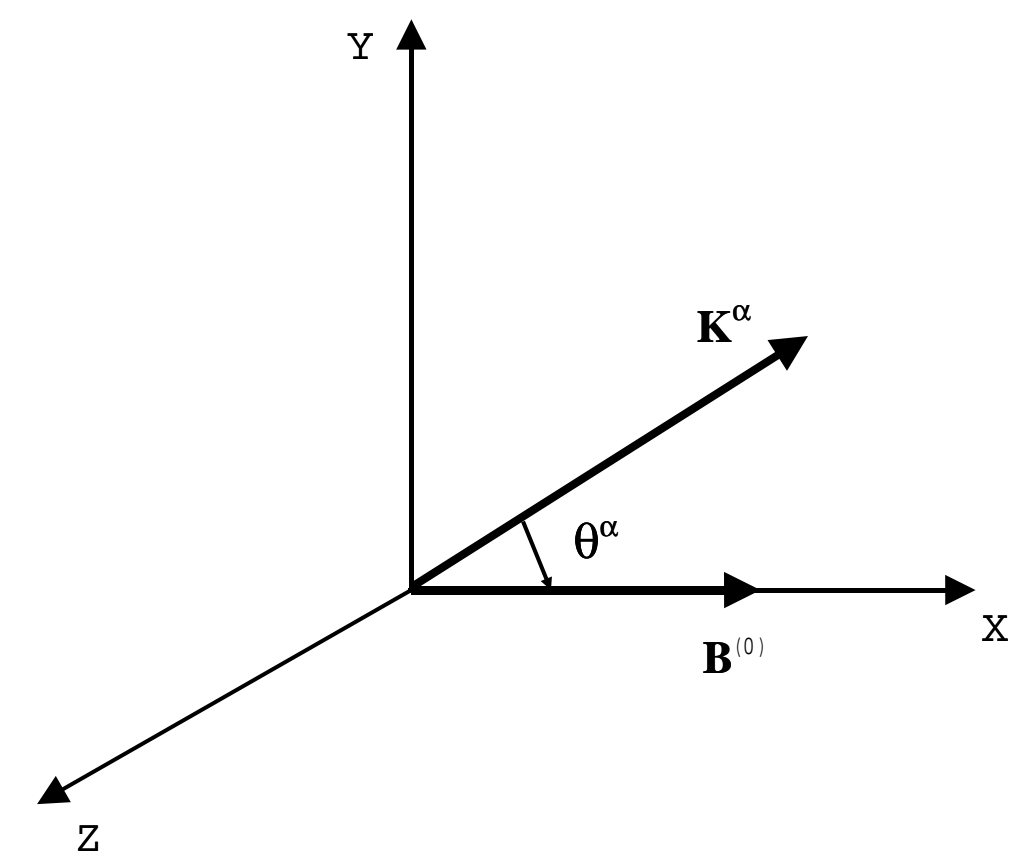

Fig. 1. Local reference frame relative to the $\alpha$ th mode.

The Eq. (26) expresses an eigenvalue problem, in which the frequency $\Omega^{\alpha}$ represents the eigenvalue, while the vector $\boldsymbol{U}_{0}^{\alpha}$ is proportional to the right eigenvector $\boldsymbol{\xi}^{\alpha}$ of the matrix $A$. We write the first-order solution in the form:

$U_{0 i}^{\alpha}=a^{\alpha} \xi_{i}^{\alpha}$

where $a^{\alpha}$ represents the complex amplitude of the $\alpha$ th mode. A straightforward calculation allows us to calculate the six eigenvalues of the matrix $A$, which are indicated in the following form:

$\Omega^{ \pm S}=0$

$\Omega^{ \pm \mathrm{A}}=\sigma c_{\mathrm{A}}^{(0)} K^{ \pm \mathrm{A}} \cos \theta^{ \pm \mathrm{A}}$

$\Omega^{ \pm \mathrm{F}}=\sigma c_{\mathrm{A}}^{(0)} K^{ \pm \mathrm{F}}$

where $\boldsymbol{c}_{\mathrm{A}}^{(0)}(\boldsymbol{x})=\boldsymbol{B}^{(0)}(\boldsymbol{x}) /\left[4 \pi \rho^{(0)}(\boldsymbol{x})\right]^{1 / 2}$ is the vector Alfvén velocity associated with the equilibrium structure, and $\sigma= \pm 1$ determines the propagation direction, parallel or antiparallel to $c_{\mathrm{A}}^{(0)}$. The vanishing frequency $\Omega^{ \pm \mathrm{S}}$ is the frequency of slow magnetosonic fluctuations, which are non-propagating in the cold plasma limit $(\beta=0) . \Omega^{ \pm \mathrm{A}}$ and $\Omega^{ \pm \mathrm{F}}$ are the frequencies of Alfvén and fast magnetosonic waves, respectively.

In calculating the eigenvectors of the slow magnetosonic mode, the condition $\nabla \cdot \boldsymbol{B}^{(1)}=0$ must be explicitly take into account. Using a similar procedure as that described above, the latter condition implies that the magnetic fluctuation at the order $O\left(\delta^{0}\right)$ must be perpendicular to the wavevector:

$B_{0 X}^{\alpha} \cos \theta^{\alpha}+B_{0 Y}^{\alpha} \sin \theta^{\alpha}=0$.

Using this condition, it is seen that the components of the slow magnetosonic eigenvector are all vanishing, except the density fluctuation, which is arbitrary. Actually, static density fluctuations can exist in a cold plasma, since no pressure gradients are associated with them. Choosing the first arbitrary component equal to $\rho^{(0)}$, the eigenvectors of slow magnetosonic mode can be written in the form

$\boldsymbol{\xi}^{ \pm \mathrm{S}}=\left(\rho_{0}^{ \pm \mathrm{S}}, 0,0,0,0,0\right)$.

In a similar way, the eigenvectors of the Alfvénic mode can be calculated, finding:

$\boldsymbol{\xi}^{ \pm \mathrm{A}}=\left(0,0,0,0, \pm c_{\mathrm{A}}^{(0)}, B^{(0)}\right)$

where the last arbitrary component was been chosen equal to $B^{(0)}$. Finally, the eigenvectors of the fast magnetosonic mode are

$\boldsymbol{\xi}^{ \pm \mathrm{F}}=\left(-\rho^{(0)} \tan \theta^{ \pm \mathrm{F}},-B^{(0)} \tan \theta^{ \pm \mathrm{F}}, \mp \frac{c_{\mathrm{A}}^{(0)}}{\cos \theta^{ \pm \mathrm{F}}}, B^{(0)}, 0,0\right)$ 
where the fourth component has been chosen equal to $B^{(0)}$. Other useful quantities for the next development are the left eigenvectors of the Alfvénic mode $\boldsymbol{\eta}^{ \pm \mathrm{A}}$, which are defined by the relation

$\eta_{i}^{ \pm \mathrm{A}} A_{i j}=\Omega^{ \pm \mathrm{A}} \eta_{j}^{ \pm \mathrm{A}}$.

They can be written in the form:

$\boldsymbol{\eta}^{ \pm \mathrm{A}}=\left(0,0,0,0, B^{(0)}, \mp c_{\mathrm{A}}^{(0)}\right)$.

Though the expressions of right and left eigenvectors of Alfvén waves have been derived in the local reference frame $\{X, Y, Z\}$, it is useful to re-express them with respect to the global reference frame $\{x, y, z\}$. We re-define the vector of unknowns with respect to the global reference frame:

$\boldsymbol{W}_{0}^{\alpha}=\left(\rho_{0}^{\alpha}, \boldsymbol{v}_{0}^{\alpha}, \boldsymbol{B}_{0}^{\alpha}\right)=\left(\rho_{0}^{\alpha}, v_{0 x}^{\alpha}, v_{0 y}^{\alpha}, v_{0 z}^{\alpha}, B_{0 x}^{\alpha}, B_{0 y}^{\alpha}, B_{0 z}^{\alpha}\right)$.

Correspondingly, the right and left eigenvectors of Alfvén waves are given by

$\boldsymbol{\xi}^{ \pm \mathrm{A}}=\left(0, \mp c_{\mathrm{A}}^{(0)} \boldsymbol{e}^{ \pm \mathrm{A}}, B^{(0)} \boldsymbol{e}^{ \pm \mathrm{A}}\right)$

$\boldsymbol{\eta}^{ \pm \mathrm{A}}=\left(0, B^{(0)} \boldsymbol{e}^{ \pm \mathrm{A}}, \mp c_{\mathrm{A}}^{(0)} \boldsymbol{e}^{ \pm \mathrm{A}}\right)$

respectively, where $\boldsymbol{e}^{ \pm \mathrm{A}}=\boldsymbol{B}^{(0)} \times \boldsymbol{K}^{ \pm \mathrm{A}} /\left|\boldsymbol{B}^{(0)} \times \boldsymbol{K}^{ \pm \mathrm{A}}\right|$ is a unit vector perpendicular both to $\boldsymbol{B}^{(0)}$ and to $\boldsymbol{K}^{ \pm \mathrm{A}}$, directed along the Alfvén wave polarization direction.

\subsection{Ray and wavevector equations}

We now consider those trajectories in the space-time, along which the phase $S^{\alpha}(\boldsymbol{x}, t)$ of the $\alpha$ th mode remains constant. These trajectories represent the rays. Using time as parameter for a given ray $x_{i}=x_{i}(t)$ the condition $S^{\alpha}(\boldsymbol{x}, t)=$ const. is expressed by

$\frac{\partial S^{\alpha}}{\partial t}+\frac{\mathrm{d} x_{j}}{\mathrm{~d} t} \frac{\partial S^{\alpha}}{\partial x_{j}}=0$

which gives

$\Omega^{\alpha}=K_{j}^{\alpha} \frac{\mathrm{d} x_{j}}{\mathrm{~d} t}$.

The evolution equation for the wavevector $\boldsymbol{K}^{\alpha}$ can be obtained by deriving Eq. (38) with respect to $x_{n}$ :

$\frac{\partial K_{n}^{\alpha}}{\partial t}+K_{j}^{\alpha} \frac{\partial}{\partial x_{n}}\left(\frac{\mathrm{d} x_{j}}{\mathrm{~d} t}\right)+\frac{\mathrm{d} x_{j}}{\mathrm{~d} t} \frac{\partial K_{n}^{\alpha}}{\partial x_{j}}=0$

Using the derivative along the ray $\mathrm{d} / \mathrm{d} t=\partial / \partial t+\left(\mathrm{d} x_{j} / \mathrm{d} t\right)\left(\partial / \partial x_{j}\right)$ we obtain

$\frac{\mathrm{d} K_{n}}{\mathrm{~d} t}=-K_{j} \frac{\partial}{\partial x_{n}}\left(\frac{\mathrm{d} x_{j}}{\mathrm{~d} t}\right)$.

Considering Alfvén waves, whose dispersion relation is

$\Omega^{ \pm \mathrm{A}}= \pm c_{\mathrm{Aj}}^{(0)} K_{j}$

and comparing with Eq. (39), we derive the equation of rays for Alfvén waves

$\frac{\mathrm{d} x_{j}^{ \pm \mathrm{A}}}{\mathrm{d} t}= \pm c_{\mathrm{A} j}^{(0)}$

Inserting this expression into Eq. (40) we obtain the evolution equation of the wavevector of Alfvén waves along the rays:

$\frac{\mathrm{d} K_{n}^{ \pm \mathrm{A}}}{\mathrm{d} t}=\mp \frac{\partial c_{\mathrm{A} j}^{(0)}}{\partial x_{n}} K_{j}^{ \pm \mathrm{A}}$.

The Eq. (41) indicates that the wavefronts of Alfvénic perturbation locally propagate along the equilibrium magnetic field $\boldsymbol{B}^{(0)}$, at the Alfvén speed. The Eq. (42) implies that variations of the wavevector $\boldsymbol{K}^{ \pm \mathrm{A}}$ along the rays are due to gradients of the Alfvén speed $\boldsymbol{c}_{\mathrm{A}}^{(0)}$. In a similar way, the ray equation for fast magnetosonic waves can be derived using the dispersion relation

$\Omega^{ \pm \mathrm{F}}= \pm K^{ \pm \mathrm{F}} c_{\mathrm{A}}^{(0)}= \pm c_{\mathrm{A}}^{(0)} \frac{K_{j}^{ \pm \mathrm{F}} K_{j}^{ \pm \mathrm{F}}}{K^{ \pm \mathrm{F}}}$. 
Comparing with Eq. (39), we find the ray equation for the fast magnetosonic waves:

$\frac{\mathrm{d} x_{j}^{ \pm \mathrm{F}}}{\mathrm{d} t}= \pm c_{\mathrm{A}}^{(0)} \frac{K_{j}^{ \pm \mathrm{F}}}{K^{ \pm \mathrm{F}}}$

which indicates that fast magnetosonic waves locally propagates in the direction of the wavevector, at the Alfvén speed. Inserting Eq. (43) into Eq. (40), we obtain

$\frac{\mathrm{d} K_{n}^{ \pm \mathrm{F}}}{\mathrm{d} t}=\mp K_{j}^{ \pm \mathrm{F}} \frac{\partial}{\partial x_{n}}\left(c_{\mathrm{A}}^{(0)} \frac{K_{j}^{ \pm \mathrm{F}}}{K^{ \pm \mathrm{F}}}\right)$.

Indicating by $\boldsymbol{u}^{ \pm \mathrm{F}}=\boldsymbol{K}^{ \pm \mathrm{F}} / K^{ \pm \mathrm{F}}$ the unit vector in the direction of $\boldsymbol{K}^{ \pm \mathrm{F}}$, the above equation becomes

$\frac{\mathrm{d} K_{n}^{ \pm \mathrm{F}}}{\mathrm{d} t}=\mp \frac{\partial c_{\mathrm{A}}^{(0)}}{\partial x_{n}} u_{j}^{ \pm \mathrm{F}} u_{j}^{ \pm \mathrm{F}} K^{ \pm \mathrm{F}} \mp c_{\mathrm{A}}^{(0)} K^{ \pm \mathrm{F}} u_{j}^{ \pm \mathrm{F}} \frac{\partial u_{j}^{ \pm \mathrm{F}}}{\partial x_{n}}$.

Since $u_{j}^{ \pm \mathrm{F}} u_{j}^{ \pm \mathrm{F}}=1$, the last term vanishes, being $u_{j}^{ \pm \mathrm{F}}\left(\partial u_{j}^{ \pm \mathrm{F}} / \partial x_{n}\right)=\left(\partial / \partial x_{n}\right)\left(u_{j}^{ \pm \mathrm{F}} u_{j}^{ \pm \mathrm{F}} / 2\right)=0$. Thus, we finally obtain the evolution equation for the wavevector of fast magnetosonic waves:

$\frac{\mathrm{d} K_{n}^{ \pm \mathrm{F}}}{\mathrm{d} t}=\mp \frac{\partial c_{\mathrm{A}}^{(0)}}{\partial x_{n}} K^{ \pm \mathrm{F}}$

\section{3. $\operatorname{Order} \delta^{0}$}

We consider the Eqs. (9)-(11) at the next order in $\delta$. Terms of order $\delta^{0}$ in Eq. (9) gives:

$\sum_{\alpha}\left[\frac{\partial \rho_{0}^{\alpha}}{\partial t}-i \Omega^{\alpha} \rho_{1}^{\alpha}+v_{0 n}^{\alpha} \frac{\partial \rho^{(0)}}{\partial x_{n}}+\rho^{(0)}\left(\frac{\partial v_{0 n}^{\alpha}}{\partial x_{n}}+i K_{n}^{\alpha} v_{1 n}^{\alpha}\right)\right] \exp \left(\frac{i S^{\alpha}}{\delta}\right)=0$

Following the same procedure as at the lower order, we multiply the Eq. (45) by the factor $\exp \left(-i S^{\beta} / \delta\right)$ and average over the intermediate scale $\ell$, thus obtaining

$\left[\frac{\partial \rho_{0}^{\alpha}}{\partial t}+v_{0 n} \frac{\partial \rho_{0}}{\partial x_{n}}+\rho_{0} \frac{\partial v_{0 n}}{\partial x_{n}}\right]+i\left[-\Omega^{\alpha} \rho_{1}^{\alpha}+\rho^{(0)} k_{n}^{\alpha} v_{1 n}^{\alpha}\right]=0$

where quantities at the order 0 and 1 have been separately grouped. In the same way, from Eqs. (10) and (11), we obtain, respectively

$\left[\frac{\partial v_{0 i}^{\alpha}}{\partial t}+\frac{1}{4 \pi \rho^{(0)}}\left(\frac{\partial B_{n}^{(0)}}{\partial x_{i}}-\frac{\partial B_{i}^{(0)}}{\partial x_{n}}\right) B_{0 n}^{\alpha}+\frac{B_{n}^{(0)}}{4 \pi \rho^{(0)}}\left(\frac{\partial B_{0 n}^{\alpha}}{\partial x_{i}}-\frac{\partial B_{0 i}^{\alpha}}{\partial x_{n}}\right)+\frac{\tilde{\eta}_{\mathrm{N}}}{\rho^{(0)}} v_{0 i}^{\alpha} K_{n}^{\alpha} K_{n}^{\alpha}\right.$

$\left.+\left(\tilde{\zeta}_{\mathrm{N}}+\frac{\tilde{\eta}_{\mathrm{N}}}{3}\right) \frac{1}{\rho^{(0)}} v_{0 n}^{\alpha} K_{n}^{\alpha} K_{i}^{\alpha}\right]+i\left[-v_{1 i}^{\alpha} \Omega^{\alpha}+\frac{B_{n}^{(0)}}{4 \pi \rho^{(0)}}\left(B_{1 n}^{\alpha} K_{i}^{\alpha}-B_{1 i}^{\alpha} K_{n}^{\alpha}\right)\right]=0$

and

$\left[\frac{\partial B_{0 i}^{\alpha}}{\partial t}+v_{0 n}^{\alpha} \frac{\partial B_{i}^{(0)}}{\partial x_{n}}-B_{n}^{(0)} \frac{\partial v_{0 i}^{\alpha}}{\partial x_{n}}+B_{i}^{(0)} \frac{\partial v_{0 n}^{\alpha}}{\partial x_{n}}+\tilde{\lambda}_{\mathrm{N}} B_{0 i}^{\alpha} k_{n}^{\alpha} k_{n}^{\alpha}\right]+i\left[-B_{1 i}^{\alpha} \Omega^{\alpha}-\left(B_{n}^{(0)} v_{1 i}^{\alpha}-B_{i}^{(0)} v_{1 n}^{\alpha}\right) K_{n}^{\alpha}\right]=0$

Equations (46)-(48) will be used to derive an equation for the amplitude $a^{ \pm \mathrm{A}}$ of the Alfvén waves. Considering Alfvén waves, the corresponding quantities at the order $\delta^{0}$ can be expressed in terms of the amplitude $a^{ \pm \mathrm{A}}$ and of the right eigenvector $\boldsymbol{\xi}^{ \pm \mathrm{A}}$ using the relations (28) and (36). Equations (46)-(48) become:

$\left[\mp c_{\mathrm{A}}^{(0)} a^{ \pm \mathrm{A}} e_{n}^{ \pm \mathrm{A}} \frac{\partial \rho^{(0)}}{\partial x_{n}} \mp \rho^{(0)} \frac{\partial}{\partial x_{n}}\left(c_{\mathrm{A}}^{(0)} a^{ \pm \mathrm{A}} e_{n}^{ \pm \mathrm{A}}\right)\right]+i\left[-\rho_{1}^{ \pm \mathrm{A}} \Omega^{ \pm \mathrm{A}}+\rho^{(0)} v_{1 n}^{ \pm \mathrm{A}} K_{n}^{ \pm \mathrm{A}}\right]=0$.

$\left\{\mp \frac{\partial}{\partial t}\left(c_{\mathrm{A}}^{(0)} a^{ \pm \mathrm{A}} e_{i}^{ \pm \mathrm{A}}\right)+\frac{1}{4 \pi \rho^{(0)}}\left(\frac{\partial B_{n}^{(0)}}{\partial x_{i}}-\frac{\partial B_{i}^{(0)}}{\partial x_{n}}\right) B^{(0)} a^{ \pm \mathrm{A}} e_{n}^{ \pm \mathrm{A}}+\frac{B_{n}^{(0)}}{4 \pi \rho^{(0)}}\left[\frac{\partial}{\partial x_{i}}\left(B^{(0)} a^{ \pm \mathrm{A}} e_{n}^{ \pm \mathrm{A}}\right)-\frac{\partial}{\partial x_{n}}\left(B^{(0)} a^{ \pm \mathrm{A}} e_{i}^{ \pm \mathrm{A}}\right)\right]\right.$

$\left.\mp \frac{\tilde{\eta}_{\mathrm{N}}}{\rho^{(0)}} c_{\mathrm{A}}^{(0)} a^{ \pm \mathrm{A}} e_{i}^{ \pm \mathrm{A}} K_{n}^{ \pm \mathrm{A}} K_{n}^{ \pm \mathrm{A}} \mp\left(\tilde{\zeta}_{\mathrm{N}}+\frac{\tilde{\eta}_{\mathrm{N}}}{3}\right) \frac{1}{\rho^{(0)}} c_{\mathrm{A}}^{(0)} a^{ \pm \mathrm{A}} e_{n}^{ \pm \mathrm{A}} K_{n}^{ \pm \mathrm{A}} K_{i}^{ \pm \mathrm{A}}\right\}+i\left[-v_{1 i}^{ \pm \mathrm{A}} \Omega^{ \pm \mathrm{A}}+\frac{B_{n}^{(0)}}{4 \pi \rho^{(0)}}\left(B_{1 n}^{ \pm \mathrm{A}} K_{i}^{ \pm \mathrm{A}}-B_{1 i}^{ \pm \mathrm{A}} K_{n}^{ \pm \mathrm{A}}\right)\right]=0$ 


$$
\begin{aligned}
& {\left[\frac{\partial}{\partial t}\left(B^{(0)} a^{ \pm \mathrm{A}} e_{i}^{ \pm \mathrm{A}}\right) \mp c_{\mathrm{A}}^{(0)} a^{ \pm \mathrm{A}} e_{n}^{ \pm \mathrm{A}} \frac{\partial B_{i}^{(0)}}{\partial x_{n}} \pm B_{n}^{(0)} \frac{\partial}{\partial x_{n}}\left(c_{\mathrm{A}}^{(0)} a^{ \pm \mathrm{A}} e_{i}^{ \pm \mathrm{A}}\right) \mp B_{i}^{(0)} \frac{\partial}{\partial x_{n}}\left(c_{\mathrm{A}}^{(0)} a^{ \pm \mathrm{A}} e_{n}^{ \pm \mathrm{A}}\right)\right.} \\
& \left.+\tilde{\lambda}_{\mathrm{N}} B^{(0)} a^{ \pm \mathrm{A}} e_{i}^{ \pm \mathrm{A}} K_{n}^{ \pm \mathrm{A}} K_{n}^{ \pm \mathrm{A}}\right]+i\left[-B_{1 i}^{ \pm \mathrm{A}} \Omega^{ \pm \mathrm{A}}-\left(B_{n}^{(0)} v_{1 i}^{ \pm \mathrm{A}}-B_{i}^{(0)} v_{1 n}^{ \pm \mathrm{A}}\right) K_{n}^{ \pm \mathrm{A}}\right]=0 .
\end{aligned}
$$

The scalar product of the Eqs. (49)-(51) with the left eigenvector (37) of Alfvén waves gives the equation

$$
\begin{aligned}
& \left\{\mp B^{(0)} e_{i}^{ \pm \mathrm{A}} \frac{\partial}{\partial t}\left(c_{\mathrm{A}}^{(0)} a^{ \pm \mathrm{A}} e_{i}^{ \pm \mathrm{A}}\right)+\frac{B^{(0)}}{4 \pi \rho^{(0)}} e_{i}^{ \pm \mathrm{A}}\left(\frac{\partial B_{n}^{(0)}}{\partial x_{i}}-\frac{\partial B_{i}^{(0)}}{\partial x_{n}}\right) B^{(0)} a^{ \pm \mathrm{A}} e_{n}^{ \pm \mathrm{A}}\right. \\
& \left.+B^{(0)} e_{i}^{ \pm \mathrm{A}} \frac{B_{n}^{(0)}}{4 \pi \rho^{(0)}}\left[\frac{\partial}{\partial x_{i}}\left(B^{(0)} a^{ \pm \mathrm{A}} e_{n}^{ \pm \mathrm{A}}\right)-\frac{\partial}{\partial x_{n}}\left(B^{(0)} a^{ \pm \mathrm{A}} e_{i}^{ \pm \mathrm{A}}\right)\right] \mp \frac{\tilde{\eta}_{\mathrm{N}}}{\rho^{(0)}} c_{\mathrm{A}}^{(0)} B^{(0)} a^{ \pm \mathrm{A}} K_{n}^{ \pm \mathrm{A}} K_{n}^{ \pm \mathrm{A}}\right\} \\
& +\left[\mp c_{\mathrm{A}}^{(0)} e_{i}^{ \pm \mathrm{A}} \frac{\partial}{\partial t}\left(B^{(0)} a^{ \pm \mathrm{A}} e_{i}^{ \pm \mathrm{A}}\right)+c_{\mathrm{A}}^{(0)^{2}} a^{ \pm \mathrm{A}} e_{i}^{ \pm \mathrm{A}} e_{n}^{ \pm \mathrm{A}} \frac{\partial B_{i}^{(0)}}{\partial x_{n}}-c_{\mathrm{A}}^{(0)} e_{i}^{ \pm \mathrm{A}} B_{n}^{(0)} \frac{\partial}{\partial x_{n}}\left(c_{\mathrm{A}}^{(0)} a^{ \pm \mathrm{A}} e_{i}^{ \pm \mathrm{A}}\right) \mp \tilde{\lambda}_{\mathrm{N}} B^{(0)} c_{\mathrm{A}}^{(0)} a^{ \pm \mathrm{A}} K_{n}^{ \pm \mathrm{A}} K_{n}^{ \pm \mathrm{A}}\right] \\
& =+i B^{(0)}\left[-v_{1 i}^{ \pm \mathrm{A}} \Omega^{ \pm \mathrm{A}} e_{i}^{ \pm \mathrm{A}}-\frac{B_{n}^{(0)}}{4 \pi \rho^{(0)}} B_{1 i}^{ \pm \mathrm{A}} K_{n}^{ \pm \mathrm{A}} e_{i}^{ \pm \mathrm{A}}\right]+i c_{\mathrm{A}}^{(0)}\left[ \pm B_{1 i}^{ \pm \mathrm{A}} \Omega^{ \pm \mathrm{A}} e_{i}^{ \pm \mathrm{A}} \pm B_{n}^{(0)} v_{1 i}^{ \pm \mathrm{A}} K_{n}^{ \pm \mathrm{A}} e_{i}^{ \pm \mathrm{A}}\right]
\end{aligned}
$$

where we used the relations $K_{i}^{ \pm \mathrm{A}} e_{i}^{ \pm \mathrm{A}}=0, B_{i}^{(0)} e_{i}^{ \pm \mathrm{A}}=0$, and $e_{i}^{ \pm \mathrm{A}} e_{i}^{ \pm \mathrm{A}}=1$. The LHS of Eq. (52) contains quantities of order $\delta^{0}$ while the RHS contains only quantities of order $\delta^{1}$. Using the equation

$B_{n}^{(0)} K_{n}^{ \pm \mathrm{A}}= \pm \Omega^{ \pm \mathrm{A}} \sqrt{4 \pi \rho^{(0)}}$

which follows from the Alfvén wave dispersion relation, it can be easily seen that the terms on the RHS of Eq. (52) vanish. Thus, quantities of order $\delta^{1}$ disappear from Eq. (52). Moreover, using the relations

$e_{i}^{ \pm \mathrm{A}} \frac{\partial e_{i}^{ \pm \mathrm{A}}}{\partial t}=\frac{1}{2} \frac{\partial}{\partial t}\left(e_{i}^{ \pm \mathrm{A}} e_{i}^{ \pm \mathrm{A}}\right)=0$

$e_{i}^{ \pm \mathrm{A}} \frac{\partial e_{i}^{ \pm \mathrm{A}}}{\partial x_{n}}=\frac{1}{2} \frac{\partial}{\partial x_{n}}\left(e_{i}^{ \pm \mathrm{A}} e_{i}^{ \pm \mathrm{A}}\right)=0$

$c_{\mathrm{A}}^{(0)}{ }^{2} B_{n}^{(0)} a^{ \pm \mathrm{A}} e_{i}^{ \pm \mathrm{A}} \frac{\partial e_{n}^{ \pm \mathrm{A}}}{\partial x_{i}}+c_{\mathrm{A}}^{(0)}{ }^{2} a^{ \pm \mathrm{A}} \frac{\partial B_{i}^{(0)}}{\partial x_{n}} e_{i}^{ \pm \mathrm{A}} e_{n}^{ \pm \mathrm{A}}=c_{\mathrm{A}}^{(0)^{2}} a^{ \pm \mathrm{A}} e_{n}^{ \pm \mathrm{A}}\left[\frac{\partial}{\partial x_{n}}\left(B_{i}^{(0)} e_{i}^{ \pm \mathrm{A}}\right)\right]=0$

and introducing the unit vector $\boldsymbol{b}^{(0)}=\boldsymbol{B}^{(0)} / B^{(0)}$ in the direction of the equilibrium magnetic field, the Eq. (52) reduces to

$\frac{\partial a^{ \pm \mathrm{A}}}{\partial t} \pm c_{\mathrm{A}}^{(0)} b_{n}^{(0)} \frac{\partial a^{ \pm \mathrm{A}}}{\partial x_{n}} \pm \frac{1}{2} \frac{c_{\mathrm{A}}^{(0)}}{B^{(0)}} a^{ \pm \mathrm{A}} b_{n}^{(0)} \frac{\partial B^{(0)}}{\partial x_{n}} \pm \frac{1}{2} a^{ \pm \mathrm{A}} b_{n}^{(0)} \frac{\partial c_{\mathrm{A}}^{(0)}}{\partial x_{n}}+\frac{1}{2}\left(\frac{\tilde{\eta}_{\mathrm{N}}}{\rho^{(0)}}+\tilde{\lambda}_{\mathrm{N}}\right) a^{ \pm \mathrm{A}} K_{n}^{ \pm \mathrm{A}} K_{n}^{ \pm \mathrm{A}}=0$

This equation represents the evolution equation for the amplitude $a^{ \pm \mathrm{A}}$ of Alfvén waves. It indicates that wave amplitude variations along the rays, defined by the Eq. (41), are due both to inhomogeneities of the equilibrium magnetic field and/or density, and to dissipation. The latter is determined both by viscosity and by resistivity.

\subsection{Energy equation}

We consider now the evolution of the perturbation energy. In the cold plasma limit the internal energy is negligible. Thus, the energy density per unit volume is defined by

$E=\frac{1}{2} \rho v^{2}+\frac{B^{2}}{8 \pi}$.

Each quantity has been decomposed as the sum of a contribution due to the equilibrium structure plus the perturbation. Inserting the expressions (5)-(7) into Eq. (54) and neglecting terms of order higher than $\varepsilon^{2}$, we find

$E=\frac{B^{(0)^{2}}}{8 \pi}+E_{\mathrm{p}}$

where the energy density due to perturbations is given by

$E_{\mathrm{p}}=\frac{\varepsilon}{4 \pi} \boldsymbol{B}^{(0)} \cdot \boldsymbol{B}^{(1)}+\varepsilon^{2}\left[\frac{1}{2} \rho^{(0)} v^{(1)^{2}}+\frac{B^{(1)^{2}}}{8 \pi}\right]$. 
The perturbation energy density varies over the shortest scale $\delta$, due to the oscillation of perturbed quantities. However, we are interested in the energy evolution at larger scales. Thus, we consider the energy density averaged over the intermediate scale $\left\langle E_{\mathrm{p}}\right\rangle_{\ell}$. Quantities relative to perturbation are decomposed as a superposition of propagating modes, according to Eqs. (13)-(15). Since $E_{\mathrm{p}}$ contains quadratic terms, we retain only the real part of each quantity, as indicated in Eqs. (13)-(15). Using the relations $\left\langle\exp \left( \pm i S^{\alpha} / \delta\right)\right\rangle_{\ell}=0$ and $\left\langle\exp \left[ \pm i\left(S^{\alpha}+S^{\beta}\right) / \delta\right]\right\rangle_{\ell}=0$, as well as the condition (21), at the lowest order $\delta^{1}$ we find

$\left\langle E_{\mathrm{p}}\right\rangle_{\ell}=\varepsilon^{2}\left[\frac{1}{2} \rho^{(0)} \sum_{\alpha} \frac{v_{0 i}^{\alpha} v_{0 i}^{\alpha *}}{2}+\frac{1}{8 \pi} \sum_{\alpha} \frac{B_{0 i}^{\alpha} B_{0 i}^{\alpha *}}{2}\right]$

where "**" indicates complex conjugate. In the above expression, the average energy density is a sum of contributions each due to a particular propagating mode. Thus, the average energy density of Alfvén waves, is given by

$\left\langle E_{\mathrm{p}}^{ \pm \mathrm{A}}\right\rangle_{\ell}=\varepsilon^{2}\left[\rho^{(0)} \frac{v_{0 i}^{ \pm \mathrm{A}} v_{0 i}^{ \pm \mathrm{A}^{*}}}{4}+\frac{B_{0 i}^{ \pm \mathrm{A}} B_{0 i}^{ \pm \mathrm{A}^{*}}}{16 \pi}\right]$.

Using the relation (28) and the form (36) of the Alfvén wave right eigenvector, we can write $v_{0 i}^{ \pm \mathrm{A}}=\mp c_{\mathrm{A}}^{(0)} a^{ \pm \mathrm{A}} e_{i}^{ \pm \mathrm{A}}$ and $B_{0 i}^{ \pm \mathrm{A}}=$ $B^{(0)} a^{ \pm \mathrm{A}} e_{i}^{ \pm \mathrm{A}}$, thus finding the expression

$\left\langle E_{\mathrm{p}}^{ \pm \mathrm{A}}\right\rangle_{\ell}=\varepsilon^{2} \frac{B^{(0)^{2}}}{8 \pi} a^{ \pm \mathrm{A}} a^{ \pm \mathrm{A}^{*}}$.

We can now derive an equation for the average energy density of Alfvén waves $\left\langle E_{\mathrm{p}}^{ \pm \mathrm{A}}\right\rangle_{\ell}$ using the Eq. (53) for the wave amplitude. We consider Eq. (53) and its complex conjugate. We multiply such two equations by a factor $\varepsilon^{2}\left(B^{(0)^{2}} / 8 \pi\right) a^{ \pm A^{*}}$ and $\varepsilon^{2}\left(B^{(0)^{2}} / 8 \pi\right) a^{ \pm \mathrm{A}}$, respectively, and we sum. Using the expression (57) and the condition $\nabla \cdot \boldsymbol{B}^{(0)}=0$, we finally obtain the equation

$\frac{\partial\left\langle E_{\mathrm{p}}^{ \pm \mathrm{A}}\right\rangle_{\ell}}{\partial t} \pm \frac{\partial}{\partial x_{n}}\left[c_{\mathrm{A}}^{(0)} b_{n}^{(0)}\left\langle E_{\mathrm{p}}^{ \pm \mathrm{A}}\right\rangle_{\ell}\right]+\left(\frac{\tilde{\eta}_{\mathrm{N}}}{\rho^{(0)}}+\tilde{\lambda}_{\mathrm{N}}\right) K_{n}^{ \pm \mathrm{A}} K_{n}^{ \pm \mathrm{A}}\left\langle E_{\mathrm{p}}^{ \pm \mathrm{A}}\right\rangle_{\ell}=0$

which describes the evolution is the space-time of the average energy density of Alfvén waves.

We consider an Alfvénic perturbation as formed by a collection of small wavepackets, each packet occupying a small volume $V(t)$. This volume moves following the wave propagation. In particular, each point in the volume $V(t)$ moves along the rays, according to Eq. (41). The energy of a given wavepacket is

$e^{ \pm \mathrm{A}}(t)=\int_{V(t)}\left\langle E_{\mathrm{p}}^{ \pm \mathrm{A}}\right\rangle_{\ell} \mathrm{d} V$.

In order to obtain an equation for $e^{ \pm \mathrm{A}}(t)$ we integrate the Eq. (58) on the volume $V(t)$ :

$\int_{V(t)}\left[\frac{\partial\left\langle E_{\mathrm{p}}^{ \pm \mathrm{A}}\right\rangle_{\ell}}{\partial t}+\frac{\partial}{\partial x_{n}}\left( \pm c_{\mathrm{A}}^{(0)} b_{n}^{(0)}\left\langle E_{\mathrm{p}}^{ \pm \mathrm{A}}\right\rangle_{\ell}\right)\right] \mathrm{d} V=-\int_{V(t)}\left(\frac{\tilde{\eta}_{\mathrm{N}}}{\rho^{(0)}}+\tilde{\lambda}_{\mathrm{N}}\right) K^{ \pm \mathrm{A}^{2}}\left\langle E_{\mathrm{p}}^{ \pm \mathrm{A}}\right\rangle_{\ell} \mathrm{d} V$.

Since all the points in $V(t)$ moves at the local Alfvén speed $\pm c_{\mathrm{A}}^{(0)} \boldsymbol{b}^{(0)}$, the RHS of Eq. (60) represents the derivative of the packet energy along the rays:

$\frac{\mathrm{d}}{\mathrm{d} t} \int_{V(t)}\left\langle E_{\mathrm{p}}^{ \pm \mathrm{A}}\right\rangle_{\ell} \mathrm{d} V=\int_{V(t)}\left[\frac{\partial\left\langle E_{\mathrm{p}}^{ \pm \mathrm{A}}\right\rangle_{\ell}}{\partial t}+\frac{\partial}{\partial x_{n}}\left( \pm c_{\mathrm{A}}^{(0)} b_{n}^{(0)}\left\langle E_{\mathrm{p}}^{ \pm \mathrm{A}}\right\rangle_{\ell}\right)\right] \mathrm{d} V$.

Thus, we obtain the equation

$\frac{\mathrm{d} e^{ \pm \mathrm{A}}}{\mathrm{d} t}=-\left(\frac{\tilde{\eta}_{\mathrm{N}}}{\rho^{(0)}}+\tilde{\lambda}_{\mathrm{N}}\right) K^{ \pm \mathrm{A}^{2}} e^{ \pm \mathrm{A}}$

where $\rho^{(0)}$ and $K^{ \pm \mathrm{A}}$ are typical values of density and normalized wavevector for the packet. In the limit of infinitesimal volume $V(t)$, the packet location at time $t$ is represented by a single point $\boldsymbol{x}(t)$, and $\boldsymbol{K}^{ \pm \mathrm{A}}(t)=\boldsymbol{K}^{ \pm \mathrm{A}}(\boldsymbol{x}(t), t)$ and $\rho^{(0)}(\boldsymbol{x}(t))$ are the normalized wavevector and the density at the packet location, respectively. The Eq. (61) describes the time evolution of energy of a small wavepacket. The RHS is negative definite; thus Eq. (61) describe energy dissipation due to both viscosity and resistivity.

It is now useful to come back to the non-normalized wavevector $\boldsymbol{k}^{ \pm \mathrm{A}}$. Using the relations $\boldsymbol{K}^{ \pm \mathrm{A}}=\delta \boldsymbol{k}^{ \pm \mathrm{A}}$ and (18), the Eqs. (41), (42) and (61) become, respectively:

$\frac{\mathrm{d} x_{i}^{ \pm \mathrm{A}}}{\mathrm{d} t}= \pm c_{\mathrm{A}}^{(0)} b_{i}^{(0)}$ 


$$
\begin{aligned}
& \frac{\mathrm{d} k_{i}^{ \pm \mathrm{A}}}{\mathrm{d} t}=\mp \frac{\partial\left(c_{\mathrm{A}}^{(0)} b_{n}^{(0)}\right)}{\partial x_{i}} k_{n}^{ \pm \mathrm{A}} \\
& \frac{\mathrm{d} e^{ \pm \mathrm{A}}}{\mathrm{d} t}=-\left(\frac{\eta_{\mathrm{N}}}{\rho^{(0)}}+\lambda_{\mathrm{N}}\right) k^{ \pm \mathrm{A}^{2}} e^{ \pm \mathrm{A}} .
\end{aligned}
$$

These three equations describe the time evolution of position, wavevector and energy of a small Alfvénic wavepacket. They have been derived for a cold plasma, but they are similar to those obtained in Paper I for an incompressible plasma. More precisely, Eqs. (62)-(64) reduces to those derived for an incompressible plasma in the special case of a uniform background density $\rho^{(0)}=1$. Thus, Eqs. (62)-(64) are more general than those derived in Paper I. In fact, they describe the Alfvénic packet dynamics not only in an inhomogeneous magnetic field, but they also allow for an inhomogeneous background density.

\section{Summary and conclusions}

In this paper we considered the propagation and dissipation of Alfvénic perturbations in a 3D inhomogeneous equilibrium structure. We used the MHD equations in the cold plasma approximation, where gravity and gas pressure gradients are neglected with respect to the Laplace force. This approximation is commonly used to represent the low $\beta$ plasma of the Corona. In a compressible plasma both Alfvénic and magnetosonic perturbations can propagate. We focused on the former, since they propagate at the Alfvén speed, locally following the magnetic field lines. Thus, in chaotic regions, where nearby magnetic lines exponentially move apart, the wave pattern is stretched and small scales are built up exponentially in time. The theoretical development we used is similar to that employed in Paper I: it is assumed that the amplitude of perturbations is small with respect to the equilibrium structure; so, nonlinear effects are neglected. Then, a WKB expansion is performed, based on the assumption that the typical wavelength $\lambda$ of perturbations is small with respect to the length scale $L$ of the equilibrium structure. Such a procedure allowed us to calculate the form of the equations which describe the behavior of small Alfvénic wavepackets: in particular, the time evolution of position, wavevector and energy of each packet which form the whole perturbation. The theoretical development starting from cold plasma MHD equations is more complex than that followed for an incompressible magnetofluid in Paper I. Equations (62)-(64) indicate that: (i) a given packet propagates following magnetic field lines; (ii) the wavevector evolution is determined by spatial variations of the background Alfvén speed, both in intensity and in direction, along the packet trajectory; as a result, the wavevector $k$ tends to increase in time (Paper I); (iii) the energy of the packet is dissipated with a rate which depends on dissipative coefficients (both viscosity and resistivity), and which is proportional to the squared wavevector $k^{2}$; thus, any increase of $k$ will results in an enhanced energy dissipation rate. For instance, in regions where the equilibrium magnetic lines are chaotic, the wavevector tends to increase exponentially (Paper I), giving origin to a fast dissipation characterized by the scaling law (1).

The aim of the present paper was to derive such equations for a cold plasma, and to show that their form is similar to that for an incompressible plasma, which had been derived in Paper I. In particular, Eqs. (62)-(64) reduce to those of Paper I in the case of a uniform background density $\rho^{(0)}=1$. We can conclude that all the results derived in Paper I and in Malara et al. (2000) about the dynamics and the dissipation of Alfvénic fluctuations, which where derived in the limit of incompressibility, can be extended to a cold plasma, at least when the background density is uniform. This is an important point, since the cold plasma approximation $(\beta=0)$ is much more suitable to describe the coronal plasma (where $\beta \ll 1)$ than the limit of incompressible plasma.

The main assumption of the model is that perturbations have a wavelength $\lambda$ much smaller than the typical scale $L$ of variation of the equilibrium structure. This allowed us to use a WKB approach. In consequence of the dynamical evolution of perturbation, the wavelength tends to decrease in time; thus, if the above assumption is verified at the initial time, then it will be satisfied for all the subsequent times. The initial condition $\lambda \ll L$ indicates that the wavelengths considered in our model do not represent the main contribution to the spectrum directly produced by photospheric motions. However, one can think that in consequence of the coupling between both transverse and longitudinal inhomogeneities at scales $L$, waves at smaller wavelengths $\left(\lambda \lesssim L \sim 10^{3} \mathrm{~km}\right.$ ) are formed. This has been suggested, for instance, for waves generated by the chromospheric network activity (Axford \& McKenzie 1992). The dissipation mechanism studied here can be directly applied to such waves.

The present dissipation mechanism has been derived within the WKB approximation $(\lambda \ll L)$. In particular, this hypothesis has allowed us to decouple Alfvénic from magnetosonic perturbations. At larger wavelengths $\lambda \sim L$ one cannot any longer separate different terms into Eqs. (9)-(11) according to their ordering in powers of the quantity $\delta$. Thus, a coupling between Alfvén and magnetosonic waves would result, already at the linear level $O(\varepsilon)$. This is an interesting situation, but its study is beyond the purpose of the present work.

The second important assumption of the model is that the amplitude of perturbations is small with respect to the background equilibrium: $\delta v / c_{\mathrm{A}}^{(0)} \ll 1$, with $\delta v$ the velocity perturbation and $c_{\mathrm{A}}^{(0)}$ the background Alfvén velocity. Measures of nonthermal velocities in the Corona (Acton et al. 1981) indicate $\delta v \sim 40-60 \mathrm{~km} \mathrm{~s}^{-1}$, while it is commonly assumed $c_{\mathrm{A}}^{(0)} \sim 10^{3} \mathrm{~km} \mathrm{~s}^{-1}$. Thus, the small amplitude condition seems to be verified in the Corona. In consequence of this assumption we neglected nonlinear terms 
in the MHD equations, which could lead to a transfer of the energy of Alfvénic perturbation toward compressive modes. The efficiency of such a transfer does not depend only on the amplitude but also on the wavevector of the Alfvén wave. For instance, it has been shown (Nakariakov et al. 1997) that phase-mixing of Alfvén waves on a purely transverse inhomogeneity, and the consequent increase of magnetic pressure gradient, leads to magnetosonic wave generation, even for a small amplitude wave. A similar effect could be present also in the configuration we are considering (a 3D inhomogeneous magnetic field), since a growth of the Alfvénic perturbation wavevector is also observed. However, provided that the Alfvén wave amplitude is not strongly nonlinear $\left(\delta v / c_{\mathrm{A}}^{(0)} \lesssim 0.1\right)$, the growth of the driven compressive fluctuation during phase-mixing saturates at amplitudes much lower than that of the Alfvén wave (Botha et al. 2000; Tsiklauri et al. 2001). This suggests that also in the situation we considered (small amplitude, small wavelength Alfvén waves in a 3D inhomogeneous equilibrium), the coupling with compressive modes does not sensibly modify our results, and can be neglected.

It is worth noting that the Eqs. (62)-(64), though similar to those obtained in Paper I, are more general. In fact, contrary to Paper I, in the present develpment no assumption of homogeneous background density has been made. Then, Eqs. (62)-(64) could be used to describe Alfvén wave propagation and dissipation in a stratified atmosphere. Ruderman et al. (1998) showed that large-scale structuring due to gravity can modify the scaling law of phase-mixing by slowing down the process of small scales generation. With Eqs. (62)-(64) the effects of both density stratification and 3D magnetic field inhomogeneities can be taken into account. This will be the subject of a forthcoming paper.

Acknowledgements. This work was partially supported by the MIUR under a National Project Fund (Cofin 2000) and by the European Community within the Research Training Network "Turbulence in Space Plamas, Theory, Observation and Simulation".

\section{References}

Acton, L. W., Culhane, J. L., Wolfson, C. J., et al. 1981, ApJ, 224, L137

Axford, W. I., \& McKenzie, J. F. 1992, in Solar Wind Seven, ed. E. Marsch, \& R. Schwenn (Oxford: Pergamon), 1

Belcher, J. W., \& Davis, L. 1971, J. Geophys. Res., 76, 3534

Botha, G. J. J., Arber, T. D., Nakariakov, V. M., \& Keenan, F. P. 2000, A\&A, 363, 1186

Califano, F., Chiuderi, C., \& Einaudi, G. 1990, ApJ, 365, 757

Califano, F., Chiuderi, C., \& Einaudi, G. 1992, ApJ, 390, 560

Davila, J. M. 1987, ApJ, 317, 514

Davila, J. M. 1991, in Mechanisms of Chromospheric and Coronal Heating, ed. P. Ulmschneider, E. Priest, \& R. Rosner (New York: Springer-Verlag), 464

Hollweg, J. 1987a, ApJ, 312, 880

Hollweg, J. 1987b, ApJ, 320, 875

Kappraff, J. M., \& Tataronis, J. A. 1977, J. Plasma Phys., 18, 209

Lee, E. M., \& Roberts, B. 1986, ApJ, 301, 430

Malara, F., Veltri, P., Chiuderi, C., \& Einaudi, G. 1992, ApJ, 396, 297

Malara, F., Primavera, L., \& Veltri, P. 1996, ApJ, 459, 347

Malara, F., Petkaki, P., \& Veltri, P. 2000, ApJ, 533, 523

Mok, Y., \& Einaudi, G. 1985, J. Plasma Phys., 33, 199

Nakariakov, V. M., Roberts, B., \& Murawski, K. 1997, Sol. Phys., 175, 93

Nakariakov, V. M., Roberts, B., \& Murawski, K. 1998, A\&A, 332, 795

Petkaki, P., Malara, F., \& Veltri P. 1998, ApJ, 500, 483 (Paper I)

Ruderman, M. S., Nakariakov, V. M., \& Roberts, B. 1998, A\&A, 338, 1118

Similon, P. L., \& Sudan, R. N. 1989, ApJ, 336, 442

Steinolfson, R. S. 1985, ApJ, 295, 213

Tsiklauri, D., Arber, T. D., \& Nakariakov, V. M. 2001, A\&A, 379, 1098

Tsiklauri, D., \& Nakariakov, V. M. 2002, A\&A, 393, 321

Tsiklauri, D., Nakariakov, V. M., \& Rowlands, G. 2003, A\&A, 400, 1051

Zimbardo, G., Veltri, P., \& Malara, F. 1984, J. Plasma Phys., 32, 141

Zimbardo, G., Veltri, P., Basile, G., \& Principato, S. 1995, Phys. Plasmas, 2, 2653 